\title{
A New Method for Modeling on Concurrent System
}

\author{
Jing Guo*, Zhongwei Xu and Meng Mei
}

School of Electronics \& Information Engineering, Tongji University, Shanghai, 201804, China

\begin{abstract}
Binary decision diagrams (BDDs) are effective means to cope with complex concurrent system. But the size of BDD itself can be relatively large. We study the BDD representation of large synchronous, asynchronous and interleaved processes with communication via shared variables. Due to the features of communication, we introduce a novel representation strategy. Based on the model, we continue to model and map up the synchrony, and detect the deadlock errors.
\end{abstract}

Keywords: Formal verification, Model checking, BDD, Concurrent system.

\section{INTRODUCTION}

To model the concurrent system is a relatively difficult task due to interaction between concurrently executing processes. The computer-aid verification is a useful and wellaccepted method to solve the problem.

Model checking has proved to be a powerful for the verification of concurrent finite state system. The system is described as a model and check whether the specifications are satisfied by the model. In symbolic model checking, the transition relation is described by boolean formulas to find satisfying assignment of the formula. States are represented as sets of boolean formulas. The key is whether the boolean formulas can be efficiently expressed as binary decision diagrams (BDDs). The variables are in form of successive case distinctions. Variables ordering is useful to normal forms. To eliminate the size of BDD is vital to the system efficiently.

Related work on representation of digital circuits [1-3]. The width of circuits is the maximum number of wires, through which any cut go through netlist. It turns out that the communication aspect of BDD is responsible for size of BDD. [3] is also concerned with digital circuits. BDD tree is a appropriate structure that is smaller than BDD itself. BDD can be used to help model checking of another structure [4]. Petri nets grows exponentially in the number of states, so it present a method for representing the state space in the form of BDD. [5] focus on the variable ordering of BDD. The impact and placement of mechanism is integrated into the procedure.

There are several difficulties in modeling BDD [6]: 1) how to represent interleaved, synchronous, asynchronous execution [7] 2) the complexity of the communication between the processes [8] 3) how to plan the concurrent processes [9]. We focused on the above problems. In this paper, we propose a extension of model method to BDD. We use mutually disjoint sets of boolean variables to represent each process. Instead of on process, our analysis constructs a compositional heuristics for variable ordering for entire system. Next we show synchronization can be modeled efficiently. Already have eliminated the detail, deadlock can be find in our reduced model.

The rest of this paper is organized as follows. Section 2 presents the preliminaries. Section 3 presents the novel model of BDD. Section 4 extends the model to model the details of synchronization and extension of it for deadlocks. Section 5 outline the summary and future work.

\section{PRELIMINARIES}

Definition (Transition System) triple (Init, $\mathrm{S}, \rightarrow$ ) where Init $\subseteq \mathrm{S}$ is the set of initial state, $\rightarrow \subseteq S \times S$ is transition relation and $\mathrm{S}$ is the set of states. Every transition is labeled with formula, which is enabling condition [10].

Binary decision diagrams (BDDs) [11] are a canonical form representation for a boolean formulas, which are often substantially more compact than traditional normal forms. Its structure is a directed acyclic graph rather than a tree, and variables are ordered as one traverses the graph from root to leaf. A path from the variable to the boolean value 1 or 0 is a form of assignment.

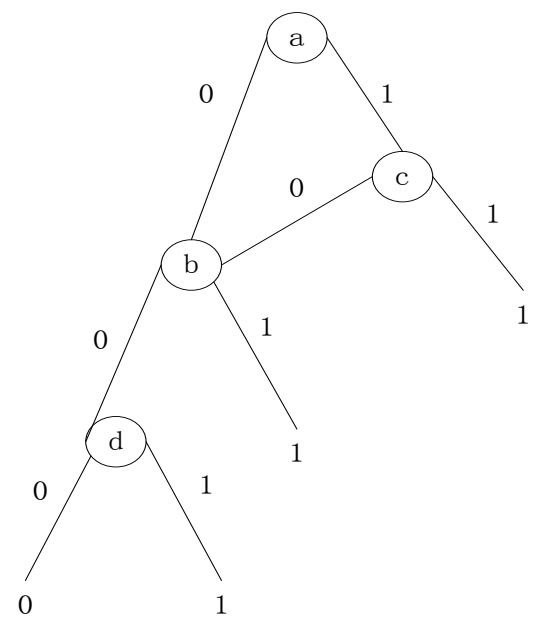

Fig. (1). A binary decision diagram. 


\section{MODELING CONCURRENT SYSTEMS WITH SHARED DATA VARIABLES}

Concurrent processes communicate each other with shared data variables. Each assignment is same as former or the value of variable is update. It is defined as follows:

$a[x:=v](y)=\left\{\begin{array}{l}a(y) \text { if } x \neq y \\ v \quad \text { if } x=y\end{array}\right.$

Particularly, $a[\varepsilon]=a$.

Every process is modeled as form of transition system (Init, $\mathrm{S}, \rightarrow$ ). However, states are in the form of $A_{p} \times L_{p}$

where $A_{p}$ is a assignment and $L_{p}$ is a location. Transitions

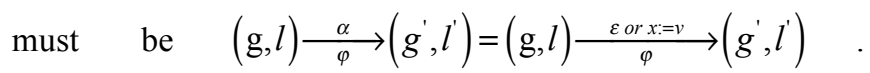
$\varepsilon$ or $x:=v$ is assignment related to shared variables, and $\varphi$ is a boolean formula. The finite set of the variables is $V_{a}$, the set of variables is $\mathrm{X} . T_{x}$ is the set of updates. Processes are indexed by i. Processes can be in form of $P_{1} \cdots, P_{i}, \cdots, P_{n}$. For $L_{i}, L_{j}$, if $i \neq j$ then $L_{i} \cap L_{j}=\varnothing$. Processes communicate each other through shared variables, so $X_{i} \cap X_{j} \neq \varnothing$. Global assignment is a function that maps the variable to a value. $g_{i}$ denoted the global assignment take place in Process i. The set of global assignment is GA. The set of indexes of processes that share $\mathrm{x}$ is given as $I n_{x}$. Processes are considered as cartesian product. If only one process is active at a time, the composition of processes is interleaving. It is a transition system $C_{1}=\left(\right.$ Init $\left., S, \rightarrow_{1}\right)$ where

1) $S=G A \times L$ (A global assignment and local location states put together a state.)

2) Init $=\left\{(g, \vec{l}) \mid\left(\left(g_{i,} \overrightarrow{l_{i}}\right) \in\right.\right.$ Init $_{i}$ for all $\left.\left.i\right)\right\}$.( In the process $i$, it is the initial state of that process.)

3) $\left(\mathrm{g}_{i}, \vec{l}_{i}\right) \underset{\varphi}{\stackrel{\alpha}{\longrightarrow}}\left(g_{i}^{\prime}, \overrightarrow{l_{i}^{\prime}}\right)$. (The interleaved global transition arises from local transition by $P_{i}$.

If some process were active at a time, the situation should be asynchronous composition. $C_{\| \mid}=\left(\right.$Init $\left., S, \rightarrow_{\|\|}\right)$where Init and $\mathrm{S}$ is same as interleaving, but the set $\operatorname{In} \in[n]$.

For each $i \in \operatorname{In},\left(\mathrm{g}_{i}, \vec{l}_{i}\right) \underset{\varphi}{\stackrel{\alpha}{\longrightarrow}}\left(g_{i}^{\prime}, \overrightarrow{l_{i}^{\prime}}\right)$.

If $i \in[n] \backslash$ In, $\vec{l}_{i}^{\prime}=\vec{l}_{i}$.
There may be some conflicts, which are processes want to update the same variable at the same time. Therefore, we map out our strategy. The conflict solution for variable $\mathrm{x}$ is a binary relation $\multimap_{x}$ between the set of $\alpha_{i} . \alpha_{i}$ must be in the process where the update exists. The relation resolve which update take place. If there were updates, all the update should meet the requirements.

If all of processes were active at a time, it would be synchrony. It is similar to synchrony apart from that $I n=[n]$. And we use $C_{\|}=\left(\right.$Init $\left., S, \rightarrow_{\|}\right)$to represent the composition.

BDDs is boolean relation, but the transition system is transition relation between states been labeled. We unify the representation by trying to use boolean formula to represent the transition.

Any variables can be a vector of $\left\{\vec{x}_{1}, \cdots, \vec{x}_{\text {length } x}\right\}$. If it updated, then $\vec{x}^{u p i}=\left\{\begin{array}{lll}\overrightarrow{u p p}^{u p} & \vec{\rightarrow}^{u p i} \\ x_{1} & , \cdots, x_{\text {lengh } h}\end{array}\right\}$. A member of vector is true, the relevant boolean vector is 1 . Otherwise, it is 0 . We define $\mathrm{X}$ as next operator, which encode state changes. LX encodes local variable update. Update flag $U_{x}^{i}$ denotes the ith transition updates $\mathrm{x}$. The $\mathrm{i}$-th transition could be transform to boolean formula as: $R\left(\rightarrow_{i}\right)=\vee(\mathrm{a}, 1) \underset{\varphi}{\stackrel{\alpha}{\longrightarrow}}\left(a^{\prime}, \mathrm{l}^{\prime}\right)$.

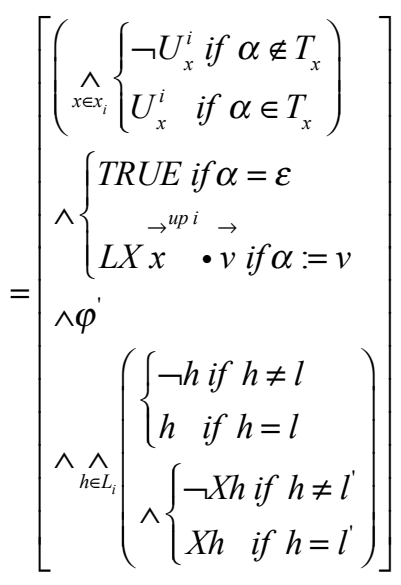

We judge whether the value is true, the related variable exist. The thinking is through the structure. In order to remark asynchronous and interleaved situation, we need to define three particular formula: idle, sched and communication. Idle describe the situation that the process remains at current location and does not carry out any variable update:

$$
\text { Idle }_{i}=\left(\underset{x \in x_{i}}{\wedge} \neg \alpha_{x}^{i}\right) \wedge\left(\underset{l \in L_{i}}{\wedge} x l \equiv l\right)
$$
time:

The second situation is only one variable is true at any 
Sched $=\underset{i=1}{\vee} \underset{j=1}{\wedge}\left\{\begin{array}{l}\neg S_{j} \text { if } j \neq i \\ s_{j} \quad \text { if } j=i\end{array}\right.$

From now on we continue to define the third situation, local copies the value from the global variable:

Communication $=\wedge \underset{x \in X}{\wedge} \underset{x \in \text { own }}{\wedge} \vec{x} \equiv \vec{x}^{u p i}$

$o w n_{i}$ is the set of index of processes where the variable update. For the conflict situation, we define the relation $\mathbb{B}_{x}$ :

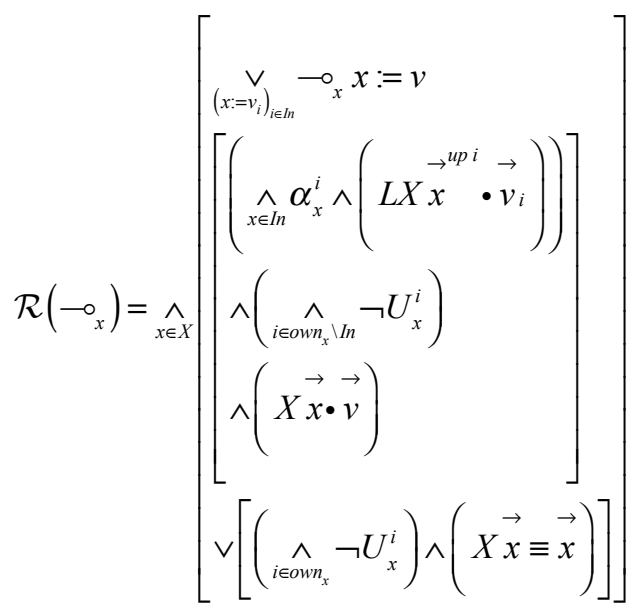

The first disjunction over all elements of $\multimap_{x}$ and the second covers the circumstance of no update.

Interleaving relation use to denote idle transition and illustrate that exactly one $s_{i}$ is true at any time.

$$
\begin{aligned}
& R\left(\rightarrow_{\mid}\right)=\left(\begin{array}{l}
\stackrel{n}{\wedge}\left(s_{i} \wedge\left(R\left(\rightarrow_{i}\right)\right) \vee\left(\neg S_{i} \wedge \text { Idle }_{i}\right)\right) \\
\wedge \text { Sched } \wedge \text { Communication } \wedge \mathcal{R}\left(\multimap_{x}\right)
\end{array}\right) \\
& R\left(\rightarrow_{\|}\right)=\left(\begin{array}{l}
\stackrel{n}{\wedge}\left(R\left(\rightarrow_{i}\right) \vee \text { Idle }_{i}\right) \\
\wedge \text { Communication } \wedge \mathcal{R}\left(\multimap_{x}\right)
\end{array}\right) \\
& R\left(\rightarrow_{\|}\right)=\left(\begin{array}{l}
{ }_{i=1}^{n} R\left(\rightarrow_{i}\right) \\
\wedge \text { Communication } \wedge \mathcal{R}\left(\multimap_{x}\right)
\end{array}\right)
\end{aligned}
$$

In interleaving, the conflict does not occur, the $\mathcal{R}\left(\multimap_{x}\right)$ merely account for local and global update.

\section{DETAILED MODEL OF SYNCHRONY}

We assume that processes communication with each other only via global variables. In real world, concurrent synchronization is a regime where multiple groups of whole system are synchronized. We establish a mechanism for model the situation better. The variable $m_{i}$ has two value state: L(locked) and U(Unlocked).

$t_{i} \cdot m_{i}=\left\{\begin{array}{l}t_{i} \text { if } m_{i}=L \\ \neg t_{i} \text { if } m_{i}=U\end{array}\right.$

If we model the process explicitly, the result would be a complicated model. Fortunately, we neglect the details. When it ignores the internal implementation, two executions have the same states: $\pi \approx_{m} \pi^{\prime}$.

$$
D=\wedge \wedge_{i \in I_{n}}\left(t_{i} \cdot m_{i} \wedge X\left(t_{i} \cdot m_{i}\right)\right)
$$

Lock wait $\mathrm{m}$ is unlocked, and changes the state. Unlock is similar.

Conditions are classified into three: wait, signal and broadcast. Signal awakens one of executions that are waiting for this condition. Broadcast awakens all of the executions that are wait for this condition. Wait use m(Locked, Unlocked) as parameter. cond[i] flag is true, there is a wait in ith process.

$$
\begin{aligned}
& \text { wait }=\left(\text { cond }[i]=1 \wedge s_{i} \bullet m_{i} \Rightarrow s_{i}\right) \wedge \\
& \left((\text { Idle until con }[i]==0) \wedge s_{i} \bullet m_{i}=s_{i}\right) \\
& \text { signal }= \\
& (\text { choose } i) \wedge\left(\begin{array}{l}
((\text { cond }[i]==1) \vee(\text { cond }==0)) \\
\Rightarrow \text { cond }[i]=0
\end{array}\right)
\end{aligned}
$$

Deadlock may be take place all the processes are waiting. Based on lock and wait, we add a global variable $P_{-}$in wait to counter the number of processes in a wait state. We want to lock the process then find the process is already in state Locked. We increase the variable. $P$ in wait is not more than N. Otherwise, a deadlock is detected. After we set up the cond, increase or decrease the $P_{-}$in wait. We use dd to illustrate that the deadlock is founded.

$$
\begin{aligned}
& \text { lock }_{\text {new }}= \\
& d d \wedge\left(\begin{array}{l}
\left(\left(t_{i}==U\right) \Rightarrow\left(t_{i} \cdot m_{i}=t_{i}\right)\right) \vee \\
\left(\left(t_{i}==L\right) \Rightarrow P_{-} \text {in_wait } \uparrow\right)
\end{array}\right) \\
& \wedge\left(\left(\text { wait }_{i}==L\right) \wedge\left(P_{-} \text {in_wait }_{-}=N \Rightarrow d d=1\right)\right) \\
& \text { wait }_{\text {new }}= \\
& d d \wedge \text { cond }[i]=1 \wedge t_{i}==U \wedge P_{-} \text {in_wait } \uparrow \\
& \wedge\left(P_{-} \text {in_wait }==N_{-} \Rightarrow \text { dd }=1\right) \\
& \wedge\left(\text { cond }[i]==0 \Rightarrow P_{-} \text {in_wait } \downarrow\right)
\end{aligned}
$$




\section{CONCLUSION}

In summary, we present a new model of interleaved, synchronous and asynchronous concurrent systems. This model shows improvement in the size. To better model synchronization, a mechanism is introduced. We hold the new trends of the concurrent processes, and plan the execution, so can detect the deadlock. For further work, we will discuss other "regular" errors and calculate the up bounds for BDDs sizes.

\section{CONFLICT OF INTEREST}

The authors confirm that this article content has no conflict of interest.

\section{ACKNOWLEDGEMENTS}

The work is support by the National Natural Science Foundation of China under grant No. 60674004, No. 61075002, the National "Twelfth Five-Year" Plan for Science \& Technology Support under grant No.2011BAG01B03, the railway ministry basic research program of China under grant No.2012AA112801, the National High Technology Research and Development Program 863 under grant No. 2012AA112801

\section{REFERENCES}

R. Bryant, "Symbolic boolean manipulation with ordered binary decision diagrams", ACM Computing Surveys, vol. 41, pp. 686698, June 1992.
[2] K. McMillan, "Symbolic Model Checking: An Approach to the State Explosion Problem," Technical Report CMU-CS-92-131, PhD Thesis, Carnegie Mellon University, Pittsburgh, Pennsylvania, USA, 1992.

[3] K. McMillan, Hierarchical Representations of Discrete Functions, with Application to Model Checking. In Computer Aided Verification, LNCS: Springer-Verlag, Germany, pp. 41-54, 1994.

[4] P. Miczulski, "Calculating State Spaces of Hierarchical Petri Nets Using BDD", In: Design of Embedded Control Systems, 2005, pp. 85-94

[5] R. Goré, and J. Thomson, "An improved BDD method for intuitionistic propositional logic: BDDIntKt system description", Lecture Notes in Computer Science, vol. 7898, pp. 275-281, 2013.

[6] T. K. Nguyen, J. Sun, Y. Liu, J. S. Dong, and Y. Liu, "Improved BDD-based discrete analysis of timed systems", Lecture Notes in Computer Science, vol. 7436, pp. 326-340, 2012.

[7] J. Sun, Y. Liu, J. S. Dong, Y. Liu, L. Shi, and E. Andr'e, "Modeling and verifying hierarchical real-time systems using stateful timed CSP", ACM Transactions on Software Engineering and Methodology, vol. 22, no. 1, pp. 3.1-3.29, 2013.

[8] J. Sun, Y. Liu, J. S. Dong, and J. Pang, "PAT: Towards flexible verification under fairness", Lecture Notes in Computer Science, vol. 5643 of Springer, pp.709-714, 2009.

[9] J. Sun, Y. Liu, J. S. Dong, and X. Zhang, “ Verifying stateful timed CSP using implicit clocks and zone abstraction", Lecture Notes in Computer Science, vol. 5885, pp. 581-600, 2009.

[10] F. Herbreteau, B. Srivathsan, and I. Walukiewicz, "Efficient emptiness check for timed b"uchi automata", Lecture Notes in Computer Science, vol. 6174, pp. 148-161, 2010.

[11] D. Beyer, C. Lewerentz, A. Noack, " Rabbit: A tool for BDD-based verification of real-time systems", Lecture Notes in Computer Science, vol. 2725, pp. 122-125, 2003.

\footnotetext{
Received: September 16, 2014

Revised: December 23, 2014

Accepted: December 31, 2014

(C) Guo et al.; Licensee Bentham Open.

This is an open access article licensed under the terms of the Creative Commons Attribution Non-Commercial License (http://creativecommons.org/licenses/by-nc/3.0/) which permits unrestricted, non-commercial use, distribution and reproduction in any medium, provided the work is properly cited.
} 